\title{
Discovery of a cluster of galaxies behind the Milky Way: X-ray and optical observations ${ }^{\star}$
}

\author{
R. Lopes de Oliveira ${ }^{1,2}$, G. B. Lima Neto ${ }^{1}$, C. Mendes de Oliveira ${ }^{1}$, E. Janot-Pacheco ${ }^{1}$, and C. Motch ${ }^{2}$ \\ 1 Instituto de Astronomia, Geofísica e Ciências Atmosféricas, Universidade de São Paulo, R. do Matão 1226, \\ 05508-900 São Paulo, Brazil \\ e-mail: rlopes@astro.iag.usp.br \\ 2 Observatoire Astronomique, UMR 7550 CNRS, 11 rue de l'Université, 67000 Strasbourg, France
}

Received 23 May 2006 / Accepted 12 July 2006

ABSTRACT

\begin{abstract}
We report the discovery of $\mathrm{Cl} 2334+48$, a rich cluster of galaxies in the Zone of Avoidance, identified in public images from the XMM-Newton archive. We present the main properties of this cluster using the XMM-Newton X-ray data, along with new optical spectroscopic and photometric observations. $\mathrm{Cl} 2334+48$ is located at $z=0.271 \pm 0.001$, as derived from the optical spectrum of the brightest member galaxy. Such redshift agrees with a determination from the X-ray spectrum $\left(z=0.263_{-0.010}^{+0.012}\right)$, in which an intense emission line is matched to the rest wavelength of the $\mathrm{Fe} \mathrm{K} \alpha$ complex. Its intracluster medium has a plasma temperature of $4.92_{-0.48}^{+0.50} \mathrm{keV}$, a sub-solar abundance of $0.38 \pm 0.12 Z_{\odot}$, and a bolometric luminosity of $3.2 \times 10^{44} \mathrm{erg} \mathrm{s}^{-1}$. A density contrast $\delta=2500$ is reached in a radius of $0.5 h_{70}^{-1} \mathrm{Mpc}$, and the corresponding enclosed mass is $1.5 \times 10^{14} M_{\odot}$. Optical images show an enhancement of $g^{\prime}-i^{\prime}>2.5$ galaxies around the central galaxy, as expected if these were cluster members. The central object is a luminous E-type galaxy, which is displaced $\sim 40 h_{70}^{-1} \mathrm{kpc}$ from the cluster X-ray centre. In addition, it has a neighbouring arc-like feature $\left(\sim 22^{\prime \prime}\right.$ or $90 h_{70}^{-1} \mathrm{kpc}$ from it), probably due to strong gravitational lensing. The discovery of $\mathrm{Cl} 2334+48$ emphasises the remarkable capability of the XMM-Newton to reveal new clusters of galaxies in the Zone of Avoidance.
\end{abstract}

Key words. galaxies: clusters: individual: XMMU J233402.7+485108 - galaxies: intergalactic medium - X-ray: galaxies: clusters

\section{Introduction}

Rich clusters of galaxies have been successfully used as tracers of large-scale structure formation and evolution, which has allowed setting constraints on various cosmological parameters (see, e.g., Bardeen et al. 1986; Henry 2000; Rosati et al. 2002). In the past, the majority of rich clusters was first identified in the optical and later observed in X-rays. However, with the advent of large and deep X-ray surveys, X-ray observations have become one of the most useful techniques for discovering clusters of galaxies, especially for intermediate and high-redshift systems (e.g. Gioia \& Luppino 1994; Rosati et al. 1998; Adami et al. 2000). On the other hand, X-rays may also be useful for detecting clusters near the Galactic plane, where the increasing number of stars and extinction makes the optical identification of background galaxies difficult (see, e.g., Kraan-Korteweg \& Juraszek 2000, and references therein).

The mapping of the large-scale structures hidden by the Galaxy has profound cosmological implications, as can be exemplified by the discovery of the Great Attractor at $(l, b) \sim$ $\left(320^{\circ}, 0^{\circ}\right)$ (Lynden-Bell et al. 1988; Kolatt et al. 1995). Indeed, the mass distribution derived from the large-scale distribution of galaxies and from the peculiar velocity field of nearby galaxies and/or clusters is still a matter of debate (see, e.g., Tonry et al. 2000; Hudson et al. 2004; Mieske et al. 2005). It is still not clear whether or not the bulk flow of galaxies is due mainly to

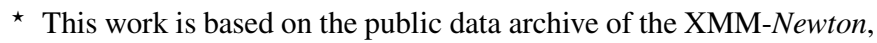
an ESA science mission with instruments and contributions directly funded by ESA member states and the USA (NASA). the Great Attractor, or if there is a large contribution from the Shapley supercluster or other more distant large-scale structures (Nagayama et al. 2006; Proust et al. 2006; Radburn-Smith et al. 2006).

A systematic search for clusters of galaxies in the Zone of Avoidance, $|b|<20^{\circ}$, was carried out by Ebeling et al. (2002), where they made use of the ROSAT All Sky Survey Bright Source Catalog (RASS-BSC Ebeling et al. 1996; Voges et al. 1999) coupled with optical and near infrared follow-ups. A major difficulty with this approach is the low-energy band of RASS (0.1-2.4 keV) in which X-rays are strongly affected by the Galactic absorption, notably when $N_{\mathrm{H}} \gtrsim 10^{21} \mathrm{~cm}^{-2}$.

In this work we report the discovery of $\mathrm{Cl} 2334+48$, a rich cluster of galaxies with $z=0.271$, projected onto the Galactic plane and identified in the XMM-Newton data archive. The source is described in Sect. 2. The main properties of this object were determined through the analysis of XMM-Newton X-ray data (described in Sect. 3.1), complemented by optical data (Sect. 3.2). The X-ray analysis is shown in Sect. 4, followed by a mass determination in Sect. 5. The optical data analysis is described in Sect. 6. For distances and luminosities, we use a $\Lambda$ CDM cosmology with $\Omega_{M}=0.3, \Omega_{\Lambda}=0.7$, and $H_{0}=70 h_{70} \mathrm{~km} \mathrm{~s}^{-1} \mathrm{Mpc}^{-1}$.

\section{The source $\mathrm{Cl} 2334+48$}

A search in $\mathrm{NED}^{1}$ shows that there are 812 known clusters and groups (221 with measured redshift) in the Zone of Avoidance.

\footnotetext{
1 Nasa Extragalactic Database, www. ned. or
} 


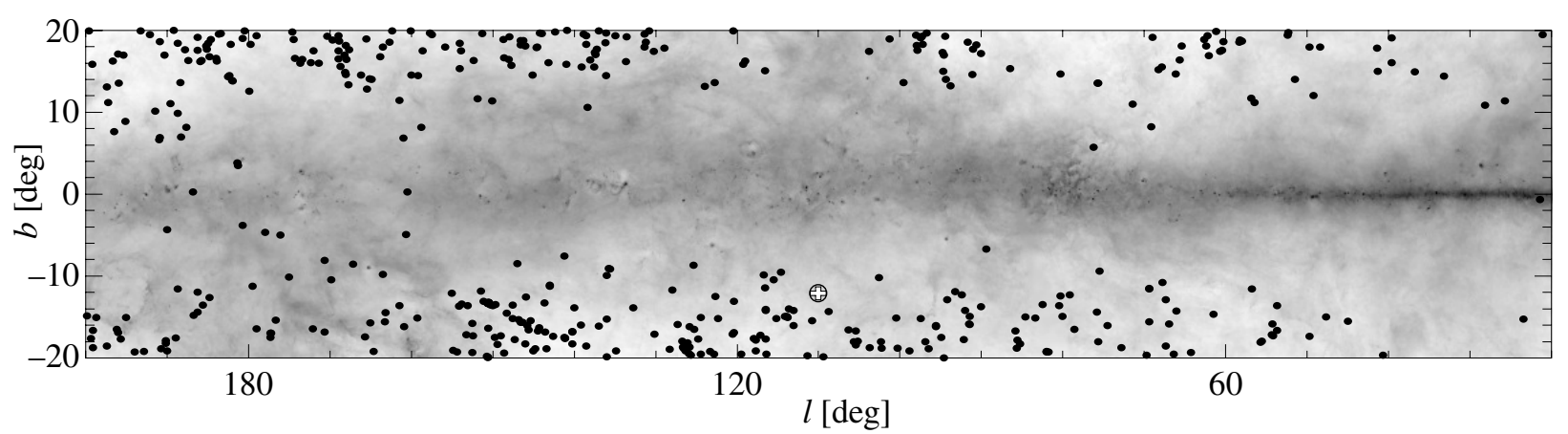

Fig. 1. Dust map $(E(B-V)$ map from Schlegel et al. (1998) in logarithmic grey scale) superposed with all known clusters and groups found in NED with $|b|<20^{\circ}$ and $20^{\circ}<l<200^{\circ}$. Cl $2334+48$ is emphasised with a larger symbol near $l=110^{\circ}, b=-12^{\circ}$.

Figure 1 shows the clusters and groups in a strip around $l=110^{\circ}$, $b=0^{\circ}$ superposed on the dust map from Schlegel et al. (1998). The number of known clusters and groups drops to 134 (59 with known redshift) in a region limited by $|b|<12.5^{\circ}$.

Within about 2 arcmin from the well-known $\mathrm{Z}$ And symbiotic star, an X-ray source, $1 \mathrm{E} 2331.6+4834$, is located at $\mathrm{RA}=23^{\mathrm{h}} 34^{\mathrm{m}} 02.7^{\mathrm{s}}$, Dec $=+48^{\circ} 51^{\prime} 08^{\prime \prime}$ (hereafter Cl 2334+48, following the usual nomenclature), found on a Galactic plane survey with Einstein IPC (Hertz \& Grindlay 1984). Even after optical and radio follow-ups by Hertz \& Grindlay (1988) and Nelson \& Spencer (1988), respectively, the nature of this source remained unknown. This position corresponds in Galactic coordinates to $l=110.0507^{\circ}, b=-12.0743^{\circ}$, where the Galactic extinction is $E(B-V)=0.211 \mathrm{mag}$ (Schlegel et al. 1998).

This source was also observed by the ROSAT PSPC and catalogued as 1WGA J2334.0+4851, with an estimated flux of $2.57 \times 10^{-13} \mathrm{erg} \mathrm{cm}^{-2} \mathrm{~s}^{-1}$ (White et al. 2000). XMM-Newton also observed 1E $2331.6+4834$ serendipitously in 2001. The MOS and pn sensitivities and spatial resolution allowed us to identify the source (XMMU J233402.7+485108) as a rich cluster of galaxies. This is thus the second cluster discovered this way by XMM-Newton in the Zone of Avoidance; the other one was XMMU J183225.4-103645, a hot $(k T=5.8 \mathrm{keV})$, $z=0.124$, rich cluster (Nevalainen et al. 2001).

\section{Observations and data analysis}

\subsection{XMM X-ray data}

Cl 2334+48 was observed twice by XMM-Newton (EPIC), in revolutions 209 on 28 January 2001, and 276 on 11 June 2001. In both observations the symbiotic system $\mathrm{Z}$ And was the main target. About $80 \%$ of the $\sim 14$ ks of the second observation (obsID 0093552801) was lost due to high soft-proton background. We report only on results obtained from the first observation (exposure time of about 24 ks; obsID 0093552701), which was partially contaminated by solar particles. This observation was made in prime full window mode with the medium filter.

The data were reduced with the Science Analysis System (SAS) software v6.5. All EPIC data were reprocessed using the epproc and emproc tasks. The data were cleaned following standard procedure, keeping only standard event grades (patterns $0-12$ for MOS1/2 and $0-4$ for the pn; flag = 0 always). Periods of high particle background were filtered out based on $E>10 \mathrm{keV}$ band light curves. For the MOS detectors, we imposed an upper threshold of 0.4 count/s and a 1 count/s for the pn. The remaining usable exposure time was $21.2 \mathrm{ks}$ for MOS1 and MOS2, and $14.2 \mathrm{ks}$ for the pn observation.
Since this cluster is located at a very low Galactic latitude, the use of standard EPIC background files (e.g. Lumb et al. 2002) is not recommended. Therefore we estimated the background using large areas free of cluster emission on the same CCD chip as the source in each camera, after removing X-ray point sources.

The spectral analysis was performed with the X-ray package XSPEC 11.3.0. The energy channels were grouped such that each bin contained at least 25 events. In all cases, the three EPIC spectra were fitted simultaneously with the same model, but allowing a free normalization factor between the different instruments.

\subsection{Optical photometry and spectroscopy}

Cl 2334+48 optical imaging was obtained by the XMM-Newton Survey Science Center (XMM-SSC) team in the framework of the XID programme (Yuan et al. 2001; Watson et al. 2001). The $\mathrm{Z}$ And field is also part of the XMM-SSC survey of the Galactic plane (Motch et al. 2003). We report on the public data taken with the optical mosaic Wide Field Camera (WFC) on the $2.5 \mathrm{~m}$ Isaac Newton Telescope (INT) with the $g^{\prime}$ and $i^{\prime}$ (Sloan system) filters on 16 July 2001. The $g^{\prime}$ image has $600 \mathrm{~s}$ exposure and $1^{\prime \prime}$ seeing, while the $i^{\prime}$ has $1200 \mathrm{~s}$ and a seeing of $1.2^{\prime \prime}$, both observed in photometric conditions.

The pre-processed and flat-fielded mosaics have astrometric solutions that are accurate to better than $0.5^{\prime \prime}$, confirmed by us from comparison with the USNO-A2.0 catalogue (Monet et al. 1998). The positions and magnitudes of all detected objects were derived using the program SExtractor (Bertin \& Arnouts 1996). Calibration to the standard SDSS system was made with the general extinction coefficients provided by the Wide Field Survey ${ }^{2}$ (WFS). It was possible to make a star-galaxy separation down to $m_{i^{\prime}}=21 \mathrm{mag}$.

A spectrum of the brightest cluster galaxy was obtained in order to check the redshift derived from the X-ray data. The observation was acquired on 14 May 2005 at the Keck II telescope, equipped with the Echellette Spectrograph and Imager (ESI) in echelle mode. A $1.25^{\prime \prime}$ slit was used, yielding a velocity resolution of about $93 \mathrm{~km} \mathrm{~s}^{-1}$. The useful range of wavelength was $\lambda \lambda 6480-7625 \AA$ (orders 7 and 8), which corresponds to $\lambda \lambda 5100-6000 \AA$ at the galaxy rest wavelength. A redshift for the object was obtained from cross-correlation techniques with a stellar spectrum of HD 19476 (a K0III star), from the Elodie database $^{3}$ (Sect. 6).

\footnotetext{
2 http://www.ast.cam.ac.uk/ wfcsur/technical/photom/

3 http://atlas.obs-hp.fr/elodie/
} 

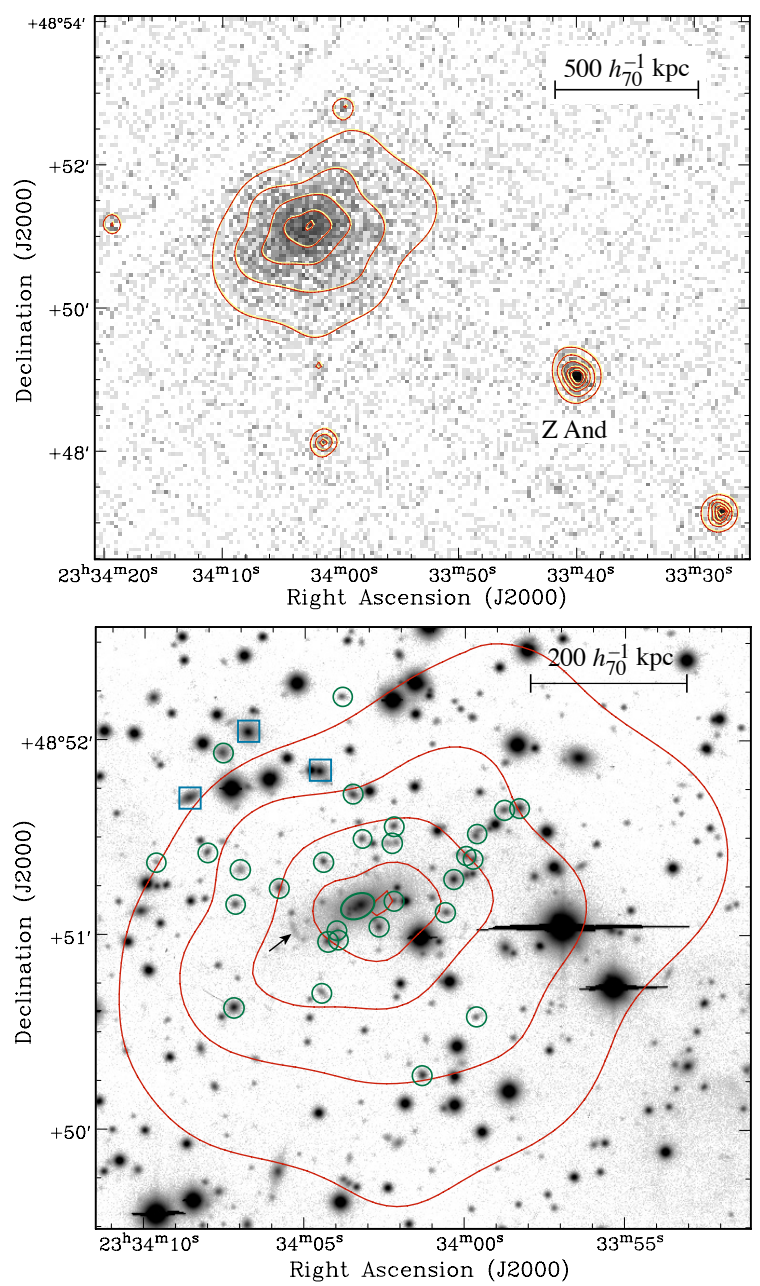

Fig. 2. Top: EPIC MOS1+MOS2 image of $\mathrm{Cl} 2334+48$ in the $0.5-8.0 \mathrm{keV}$ band. The lines are adaptively smoothed contours in logarithmic scales. Bottom: the smoothed X-ray contours superposed on the $i^{\prime}$-band image. The circles indicate the identified galaxies inside a region of radius $270 h_{70}^{-1} \mathrm{kpc}$. Squares mark the next three brightest galaxies after the first-ranked galaxy (see Sect. 6 and Fig. 10). An arc-like feature is indicated with an arrow (see Sect. 6).

\section{Global properties of the X-ray gas}

\subsection{Imaging}

Both EPIC-MOS and pn have a point spread function (PSF) with $F W H M \sim 5^{\prime \prime}$; however, their PSF have somewhat extended wings and the half energy width ${ }^{4}$ is $\sim 14^{\prime \prime}$. The PSF was not taken into account in our analysis.

The X-ray emissivity map shows that $\mathrm{Cl} 2334+48$ is quite a regular cluster. Figure 2 displays the X-ray $(0.5-8.0 \mathrm{keV}$; top panel) and $i^{\prime}$-band (bottom panel) images, both with the smoothed X-ray contours overlaid. There are a few features to note. The peak X-ray emission is displaced from the brightest cluster member by $\sim 10^{\prime \prime}$ westwards. At the cluster redshift ( $z=0.271$; see Sect. 6 ), this offset corresponds to $\sim 40 h_{70}^{-1} \mathrm{kpc}$. A number of point sources close to $\mathrm{Cl} 2334+48$ are identified with active stars and AGNs (Motch et al. 2006). The bottom panel of Fig. 2 shows the probable cluster members inside a radius of $270 h_{70}^{-1} \mathrm{kpc}$ (see details in Sect. 6).

${ }^{4}$ http://xmm.vilspa.esa.es/external/xmm_user_support/ documentation/uhb/index.html

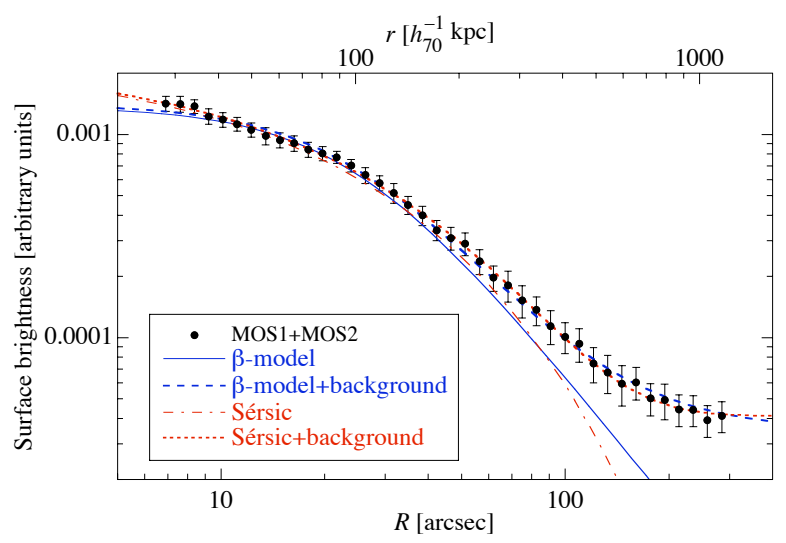

Fig. 3. X-ray surface brightness fitted with a $\beta$-model and Sérsic law (see text for details).

Table 1. Surface brightness fitting results, where $R_{\mathrm{c}}(\beta$-model) and $a$ (Sérsic profile) are in $h_{70}^{-1} \mathrm{kpc}$, and $n_{0}$ is in $10^{-2} \mathrm{~cm}^{-2}$.

\begin{tabular}{lccc}
\hline \hline Model & $R_{\mathrm{c}}$ or $a$ & $\beta$ or $v$ & $n_{0}$ \\
\hline$\beta$-model & $98.1 \pm 8.5$ & $0.520 \pm 0.022$ & $1.49 \pm 0.10$ \\
Sérsic & $65 \pm 14$ & $0.716 \pm 0.078$ & $1.30 \pm 0.12$ \\
\hline
\end{tabular}

Using the task ellipse from STSDAS/IRAF ${ }^{5}$, we derived the $\mathrm{X}$-ray brightness radial profile $I(R)$. We used a $0.5-8.0 \mathrm{keV}$ band image, combining exposure-map corrected MOS1 and MOS2 data, binned such that 1 image pixel corresponds to $6.4^{\prime \prime}$. The CCD gaps and point sources have been masked out.

In Fig. 3, we show the brightness profile, together with two different fits: a Sérsic profile (Sérsic 1968) given by

$I(R)=I_{0} \exp \left[-\left(\frac{R}{a}\right)^{v}\right]+B_{1}$

and a $\beta$-model (Cavaliere \& Fusco-Femiano 1976), in which

$I(R)=I_{0}\left[1+\left(\frac{R}{R_{\mathrm{c}}}\right)^{2}\right]^{-3 \beta+1 / 2}+B_{2}$,

where $I_{0}$ is the central surface brightness, $R_{\mathrm{c}}$ and $a$ are scale parameters, and $B_{1}$ and $B_{2}$ are constant terms to account for the background contribution. Table 1 summarises the resulting parameters.

The Sérsic profile gives a slightly better fit due to the first three surface brightness data points (the ones most strongly affected by the PSF), while the $\beta$-model flattens too much towards the centre in comparison to the peaked X-ray profile (see Fig. 3 ). Notice that the background starts to have an important effect already at $\sim 30^{\prime \prime}$ from the centre.

In order to estimate the central electronic density, $n_{0}$, which is related to $I_{0}$, we integrated the bremsstrahlung emissivity along the line-of-sight within $20^{\prime \prime}$ in the central region. The result was compared to the flux obtained by spectral fitting of the same region (the normalization parameter of the thermal spectral model in XSPEC, which is proportional to $n_{\mathrm{e}}^{2}$; Table 1).

\footnotetext{
${ }^{5}$ STSDAS is a product of the Space Telescope Science Institute, which is operated by AURA for NASA. IRAF is distributed by the NOAO, which are operated by the Association of Universities for Research in Astronomy, Inc., under cooperative agreement with the NSF.
} 
Table 2. Spectral fit results with a PHABS $*$ MEKAL model (see Sect. 4.2). Uncertainties are at the $90 \%$ confidence level.

\begin{tabular}{|c|c|c|c|c|c|c|c|}
\hline $\begin{array}{c}N_{\mathrm{H}} \\
\left(10^{21} \mathrm{~cm}^{-2}\right)\end{array}$ & $\begin{array}{c}k T \\
(\mathrm{keV})\end{array}$ & $\begin{array}{c}Z \\
\left(Z_{\odot}\right)\end{array}$ & redshift & $\begin{array}{c}f_{X}[0.5-2.0 \mathrm{keV}]^{\ddagger} \\
\left(10^{-13} \mathrm{erg} \mathrm{cm}^{-2} \mathrm{~s}^{-1}\right)\end{array}$ & $\begin{array}{c}f_{X}[2.0-10.0 \mathrm{keV}]^{\ddagger} \\
\left(10^{-13} \mathrm{erg} \mathrm{cm}^{-2} \mathrm{~s}^{-1}\right)\end{array}$ & $\begin{array}{l}\left.L_{X} \text { [bolom. }\right]^{\ddagger} \\
\left(10^{44} \mathrm{erg} \mathrm{s}^{-1}\right)\end{array}$ & $\chi_{v}^{2} /$ d.o.f. \\
\hline $1.6_{-0.1}^{+0.2}$ & $4.84_{-0.42}^{+0.50}$ & $0.40_{-0.12}^{+0.13}$ & $0.263_{-0.010}^{+0.012}$ & 4.6 & 5.8 & 3.0 & $1.09 / 246$ \\
\hline $1.39^{-0.1}$ & $5.43_{-0.38}^{+0.42}$ & $0.41_{-0.13}^{+0.12}$ & $0.264_{-0.010}^{+0.019}$ & 4.3 & 6.0 & 3.0 & $1.12 / 247$ \\
\hline $1.6_{-01}^{+0.2}$ & $4.92_{-0.58}^{+0.50}$ & $0.38_{-012}^{+0.12}$ & $0.271^{*}$ & 4.6 & 5.8 & 3.2 & $1.09 / 247$ \\
\hline $1.39^{-1.1}$ & $5.48_{-0.39}^{+0.40}$ & $0.39_{-0.12}^{+0.13}$ & $0.271^{*}$ & 4.3 & 6.0 & 3.2 & $1.12 / 248$ \\
\hline
\end{tabular}

${ }^{\ddagger}$ Corrected for absorptions; ${ }^{\dagger}$ fixed at the Galactic value (Dickey \& Lockman 1990); ${ }^{*}$ fixed at the optical redshift (Sect. 6).

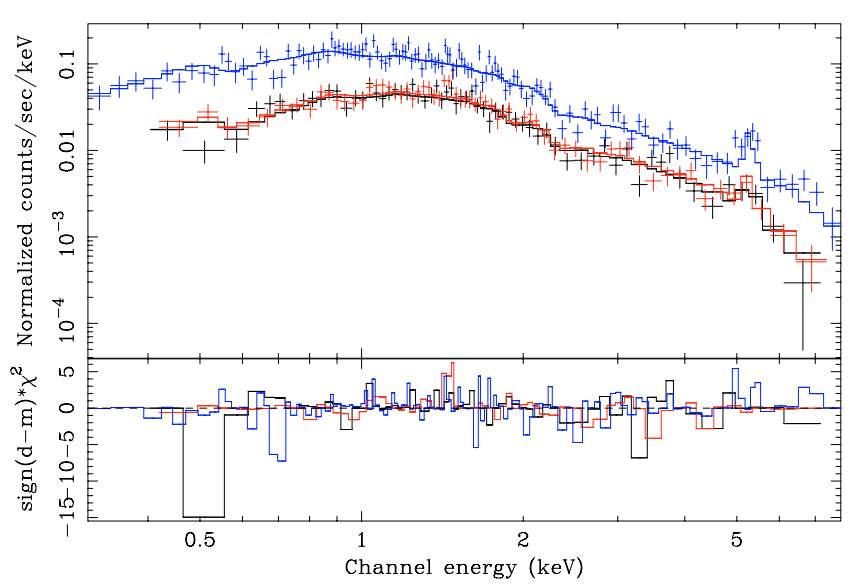

Fig. 4. EPIC spectra with the best-fit model (solid lines; see Table 2).

\subsection{Spectral analysis}

We extracted integrated EPIC spectra in the $0.3-8.0 \mathrm{keV}$ band from a circular region of $65^{\prime \prime}$ radius $\left(270 h_{70}^{-1} \mathrm{kpc}\right.$ at $\left.z=0.27\right)$, centred on the cluster X-ray image (see Fig. 4). The source's photons correspond to about $90 \%$ of the total good events ( $~ 1700$ photons in each MOS camera, and $\sim 3400$ in the pn camera), the remaining being due to background contributions.

The X-ray spectral properties of the ICM can be described very well by the MEKAL model (thermal plasma emission; Kaastra \& Mewe 1993; Liedahl et al. 1995), associated to the PHABS model (Balucinska-Church \& McCammon 1992) to account for the photoelectric absorption (Table 2). We opted for the standard Anders \& Grevesse (1989) abundance table.

A relatively strong emission line was detected at $5.27_{-0.10}^{+0.08} \mathrm{keV}$, with an equivalent width of $669_{-284}^{+315} \mathrm{eV}$. Setting all parameters free during the fit, we found that this line is compatible with the $\mathrm{Fe} \mathrm{K} \alpha$ complex emitted by a thermal $\mathrm{X}$-ray gas located at $z=0.263_{-0.010}^{+0.012}$. This fit results in a plasma temperature of $k T=4.84_{-0.42}^{+0.50} \mathrm{keV}$ and a sub-solar abundance of $Z=0.40_{-0.12}^{+0.13} Z_{\odot}$. The resulting hydrogen column density, $N_{\mathrm{H}}=1.6_{-0.1}^{+0.2} \times 10^{21} \mathrm{~cm}^{-2}$, is only slightly higher than the mean Galactic value obtained in the Dickey \& Lockman (1990) HI survey $\left(N_{\mathrm{H}} \sim 1.39 \times 10^{21} \mathrm{~cm}^{-2}\right)$. Therefore, there is no evidence of any notable local excess absorption. To verify this statement, we fitted the whole cluster by fixing the $N_{\mathrm{H}}$ to the Galactic value. The derived temperature, metal abundance, and redshift are compatible with those of the first model (see Table 2).

The redshift derived from the X-ray spectrum of $\mathrm{Cl} 2334+48$ agrees very well with the $z=0.271 \pm 0.001$ obtained from the optical spectrum of the brightest cluster member (Sect. 6). Since there are lower uncertainties in the optical redshift

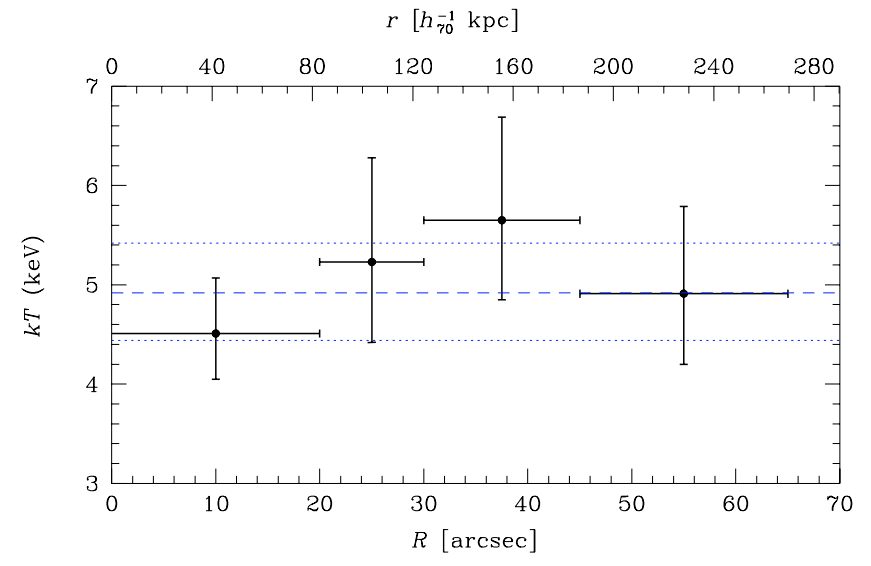

Fig. 5. Radial temperature profile (see Sect. 4.2.1). The dashed line represents the mean temperature $(k T=4.92 \mathrm{keV}$; dotted line for their limits) derived from the spectrum extract within $R<65^{\prime \prime}$. Error bars are at the $90 \%$ confidence level.

determination, this redshift was used in the X-ray spectral fits. As before, similar results are obtained if the absorption column is taken as a free parameter, or if freezing it to the Galactic value. Both results are also statistically indistinguishable from those obtained freeing the redshift in the fits.

\subsubsection{Temperature profile}

In order to determine the temperature profile, we divide the cluster image in a central circular region and three concentric rings. Their dimensions are such that the signal-to-noise ratios are approximately constant in each accumulated data set, as a compromise between the spatial resolution and the quality of the spectral fitting.

The EPIC spectra of each spatial region were fitted with the PHABS $*$ MEKAL model exactly as in Sect. 4.2, but the absorption column $\left(N_{\mathrm{H}}=1.6 \times 10^{21} \mathrm{~cm}^{-2}\right)$, metallicity $\left(Z=0.38 Z_{\odot}\right)$, and redshift $(z=0.271)$ were kept fixed to the adopted values of the integrated spectrum (Table 2 ). The resulting temperature profile is shown in Fig. 5, where we can see that it shows the characteristic rise from the centre and then falls outward. However, given the large error bars and the low spatial resolution, the temperature profile is still consistent with an isothermal profile at least up to $\sim 70^{\prime \prime}$. Hence, we adopt an isothermal profile with $k T=4.92_{-0.48}^{+0.50} \mathrm{keV}$, as derived in Sect. 4.2. 


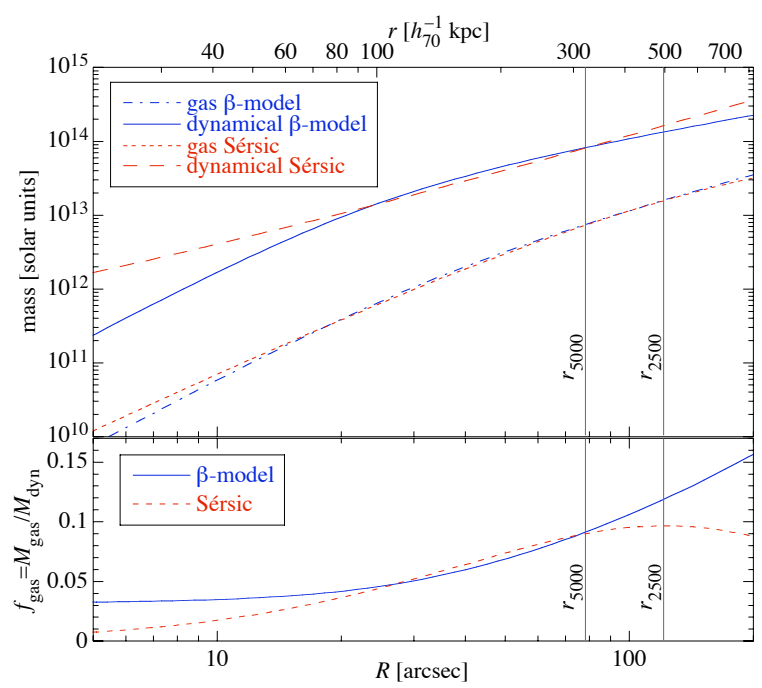

Fig. 6. Top: gas and dynamical (total) mass cumulative profiles. The vertical lines indicate the $r_{2500}$ and $r_{5000}$ radii (see text). Bottom: the gas mass fraction (baryon fraction minus the contribution from galaxies).

\section{Mass determination}

\subsection{Gas and dynamical mass}

The gas mass was obtained by simply integrating the gas density obtained in Sect. 4.1. The dynamical mass was computed with the assumption of spherical symmetry and hydrostatic equilibrium of the X-ray emitting gas, with the density and temperature profiles obtained above.

We used the $\beta$-model and Sérsic law. Both models can be integrated analytically, resulting in

$$
M(<r)=4 \pi \mu m_{\mathrm{p}} n_{0}\left\{\begin{array}{l}
\frac{a^{3}}{v} \gamma\left[\frac{3-p}{v},\left(\frac{r}{a}\right)^{v}\right] \\
\frac{r^{3}}{3}{ }_{2} F_{1}\left[\frac{3}{2}, \frac{3 \beta}{2}, \frac{5}{2},-\left(\frac{r}{r_{c}}\right)^{2}\right],
\end{array}\right.
$$

where the first line is for the Sérsic profile and the bottom line for the $\beta$-model. $\gamma(a, x)$ and ${ }_{2} F_{1}(a, b, c, x)$ are the incomplete gamma and the hypergeometric functions, respectively (see, e.g., Arfken $1970)$. For both models, $\mu$ is the mean molecular weight $(\sim 0.6$, for a fully ionized primordial plasma) and $m_{\mathrm{p}}$ is the proton mass. For the Sérsic profile, we also have $2 p=1-0.6097 v+0.05563 v^{2}$ (see Lima Neto et al. 1999; Durret et al. 2005, for the deprojection of a Sérsic profile).

Figure 6 shows the resulting gas mass profiles, together with the dynamical mass. Both models predict quite the same gas mass profile in the range $6^{\prime \prime} \lesssim R \lesssim 200^{\prime \prime}$ (corresponding to $30 \lesssim r \lesssim 800 h_{70}^{-1} \mathrm{kpc}$ ), where the data are most reliable. Beyond these limits, the gas mass profiles diverge. The total gas mass at $200^{\prime \prime}$ is either $3.2 \times 10^{13} M_{\odot}$ or $3.5 \times 10^{13} M_{\odot}$ depending on whether using the Sérsic profile or the $\beta$-model, respectively. The difference between the total mass profiles is more marked than the gas profiles, especially near the centre. At 200", the dynamical mass is either $3.8 \times 10^{14} M_{\odot}$ for the Sérsic profile or $2.3 \times 10^{14} M_{\odot}$ for the $\beta$-model.

\subsection{Virial radius and gas mass fraction}

We also computed the dynamical mass density as a function of radius, which can, for example, be compared to the density

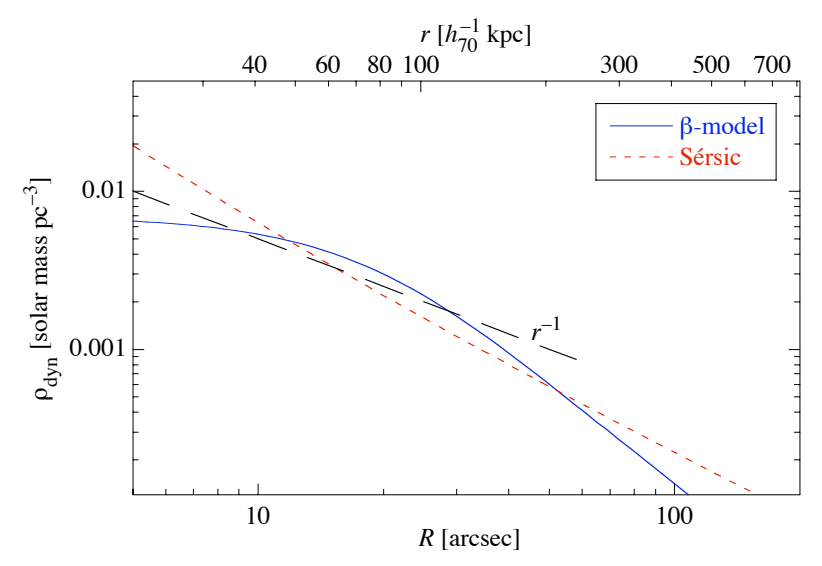

Fig. 7. Total mass density profile for both $\beta$-model and Sérsic law. The long-dashed line shows $\rho \propto r^{-1}$ as a reference.

of dark matter halos formed in cosmological $N$-body simulations. In Fig. 7 we show the dynamical mass density compared to a steep $\rho \propto r^{-1}$ profile, the same inner slope of the Navarro et al. (1997, NFW) "universal" profile for dark halos. Note that the inner slope of the total mass density is close to $\rho \propto r^{-1}$, like the NFW profile.

While the use of the Sérsic law resulted in a very steep total density profile, adopting the $\beta$-model resulted in a central flat total density profile. However, both profiles are steep up to the $\mathrm{X}$-ray image resolution; the flattening of the $\beta$-model is actually seen only when the profile is extrapolated inwards.

We estimated the radius corresponding to some values of the density contrast $\delta=\bar{\rho}\left(r_{\delta}\right) / \rho_{\mathrm{c}}(z)$. For $\delta=200$, we have the usual $r_{200}$, frequently associated with the virial radius. The available data are restricted to about $200^{\prime \prime}$, which corresponds to a ratio $\delta \sim 1000$, and therefore well inside the virial radius.

Extrapolating the derived mass profile (Eq. (3)) from the available data, we obtained $r_{200}=3.4 h_{70}^{-1} \mathrm{Mpc}$ and a corresponding virial mass of $3.8 \times 10^{15} M_{\odot}$ for the Sérsic law, or else $r_{200}=1.7 h_{70}^{-1} \mathrm{Mpc}$ and $4.8 \times 10^{14} M_{\odot}$ for the $\beta$-model. Such a large discrepancy comes from the extrapolation of two models, owing to the different asymptotical behaviours, and those values are highly uncertain.

Using the mean cluster temperature as an estimator of the virial radius (Evrard et al. 1996),

$r_{200}=2.78 h_{70}^{-1}\left(\frac{k T}{10 \mathrm{keV}}\right)^{1 / 2}\left(\Omega_{M}(1+z)^{3}+\Omega_{\Lambda}\right)^{-1 / 2}$

we obtain $r_{200}=1.7 h_{70}^{-1} \mathrm{Mpc}$. The expected mass inside $r_{200}$, which we take as the virial mass, using the relation $M_{200} \equiv$ $(4 \pi / 3) 200 \rho_{\mathrm{c}} r_{200}^{3}$ is $5 \times 10^{14} M_{\odot}$. Both values are close to that of the $\beta$-model.

For $\delta=5000$, the equivalent radius can be calculated more precisely. We obtain $r_{5000} \sim 320 h_{70}^{-1} \mathrm{kpc}$ for both models, which corresponds to an enclosed mass of $M_{5000}=8.1 \times 10^{13} M_{\odot}$. For $\delta=2500$, the radius is still within the X-ray data image, and we have $r_{2500}=0.5 h_{70}^{-1} \mathrm{Mpc}$ and $M_{2500}=1.5 \times 10^{14} M_{\odot}$. These values are very close to the ones from a similar cluster, Abell 1068, which has $r_{2500}=490 h_{70}^{-1} \mathrm{kpc}, M_{2500}=1.47 \times$ $10^{14} M_{\odot}$, and $k T=4.67 \mathrm{keV}$ (Arnaud et al. 2005). 


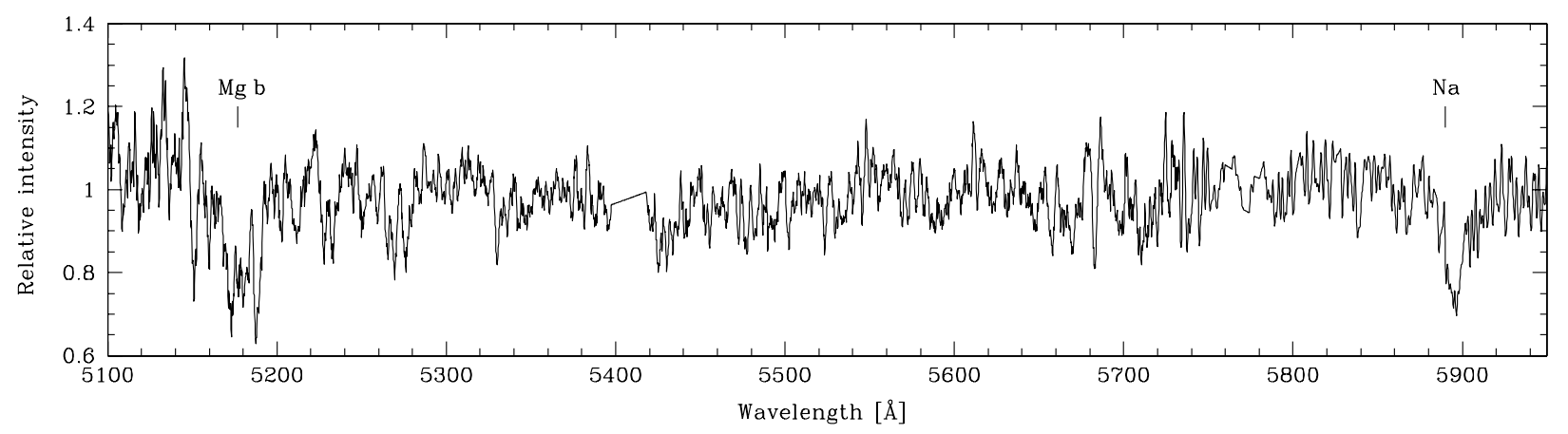

Fig. 8. The optical spectrum of the central galaxy, de-redshifted and smoothed with a boxcar filter.

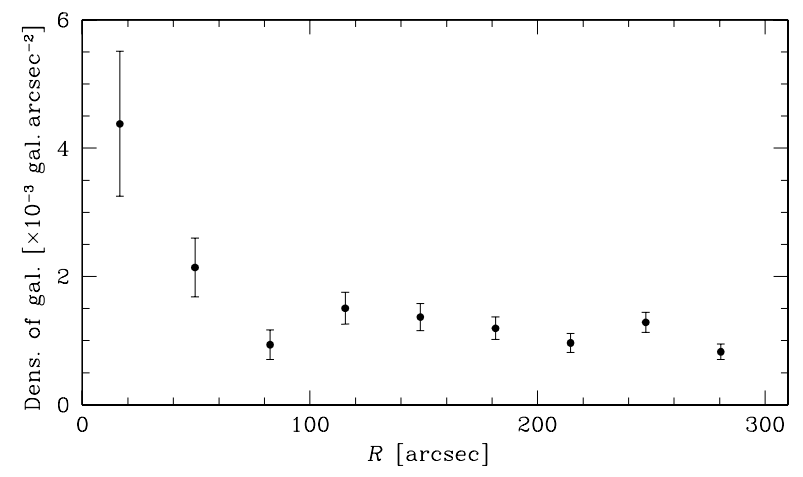

Fig. 9. Density of galaxies with $16.3<m_{i^{\prime}}<21$ as a function of the distance from the central galaxy in steps of $33^{\prime \prime}$.

The gas mass fraction, $f_{\text {gas }}$, is computed simply as the ratio between the gas mass and the total mass at a given radius, and is related to the baryon fraction as

$$
\frac{\text { baryon fraction }}{\text { gas fraction }}=1+\frac{M_{\mathrm{gal}}}{M_{\mathrm{gas}}} \text {. }
$$

Here $M_{\text {gal }}$ is the baryonic mass in galaxies, which may be estimated as $M_{\text {gal }} \sim 0.16 h_{70}^{0.5} M_{\text {gas }}$ (White et al. 1993; Fukugita et al. 1998).

Figure 6 (bottom panel) shows the obtained gas mass fraction. For the $\beta$-model, $f_{\text {gas }}$ rises up to the outer limit of the X-ray data, while this fraction rises up to $\sim r_{2500}\left(\sim 500 h_{70}^{-1} \mathrm{kpc}\right)$ and then decreases very slowly for the Sérsic model. This difference in behaviour comes from the different form of the dynamical mass for each model. At $200^{\prime \prime}, f_{\text {gas }}$ is $\sim 0.16$ for the $\beta$-model and $\sim 0.09$ for the Sérsic profile. These values are close to those obtained by Allen et al. (2002), $\bar{f}_{\text {gas }}=0.113 \pm 0.005$ at $r_{2500}$, for six regular clusters.

Within the interval $5^{\prime \prime} \lessgtr R \lesssim 200^{\prime \prime}$, the derived dynamical properties, dynamical mass, gas fraction, and total mass density profile could be considered as normal if compared to the established properties of the regular rich clusters of galaxies.

\section{Optical properties}

The redshift of the central galaxy $\left(\mathrm{RA}=23^{\mathrm{h}} 34^{\mathrm{m}} 03.2^{\mathrm{s}}\right.$, Dec $=$ $\left.+48^{\circ} 51^{\prime} 09.3^{\prime \prime}\right)$ was derived from echelle spectroscopy, as described in Sect. 3.2. In particular, the $\mathrm{Mg}_{\mathrm{b}}$ band and the $\mathrm{Na}$ lines are very prominent in the spectrum (Fig. 8). We derived a redshift $z=0.271 \pm 0.001$, which confirms the value independently obtained from the X-ray analysis.

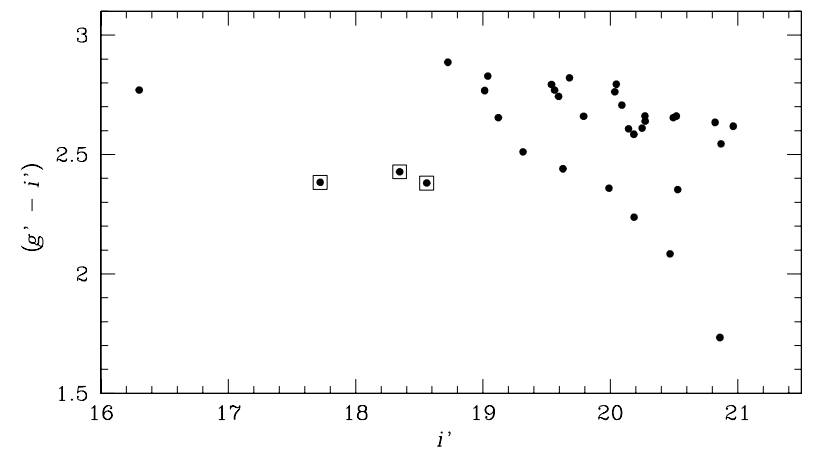

Fig. 10. Colour-magnitude diagram in the $g^{\prime}$ and $i^{\prime}$ bands inside a circle of radius $65^{\prime \prime}$, centred on the brightest cluster galaxy. The next three brightest galaxies after the first-ranked galaxy of the cluster are marked with squares (see Sect. 6).

The $g^{\prime}$ and $i^{\prime}$ images were used to estimate the extent of the cluster and obtain the colour-magnitude diagram. We show the density of galaxies with observed magnitudes $16.3<m_{i^{\prime}}<21$ in Fig. 9, in concentric annuli of increasing radii around the central galaxy (which has $m_{i^{\prime}}=16.3 \mathrm{mag}$ ). As expected, the density increases steeply towards the centre of the cluster within a radius of about $65^{\prime \prime}$ (or $270 h_{70}^{-1} \mathrm{kpc}$ at $z=0.27$ ). We then use all identified galaxies in this region to obtain the colour-magnitude diagram shown in Fig. 10. The most probable cluster members have $g^{\prime}-i^{\prime} \gtrsim 2.5$.

The colour-magnitude diagram shows that the next three brightest galaxies after the first-ranked galaxy of the cluster (Fig. 2, lower panel, and Fig. 10) have luminosities from four to six times lower than that of the central galaxy. These three objects are located in the northeastwards region. Without redshifts, we can just speculate at this point that these could form a foreground group, although they do have the right colours to be in the cluster.

We obtained the surface brightness profile of the central object with only the $i^{\prime}$ image (Fig. 11), since the $g^{\prime}$ image did not have sufficient signal-to-noise ratio. An $r^{1 / 4}$-profile was fitted to the galaxy surface brightness profile between $1.5^{\prime \prime}$ and $22^{\prime \prime}$. There is no significant light excess over the de Vaucouleurs profile, as one would expect for a cD galaxy.

An arc-like structure is identified in both the $g^{\prime}$ and $i^{\prime}$ images (see Fig. 2, lower panel). Its relative position, tangential to the semi-major axis of the expected mass distribution of the cluster, suggests that this feature could be due to strong gravitational lensing. Such an arc-like feature is compatible with what is expected for a distant galaxy at $z>1$, lensed by a cluster with mass similar to that derived for $\mathrm{Cl} 2334+48$ in Sect. 5.1. 


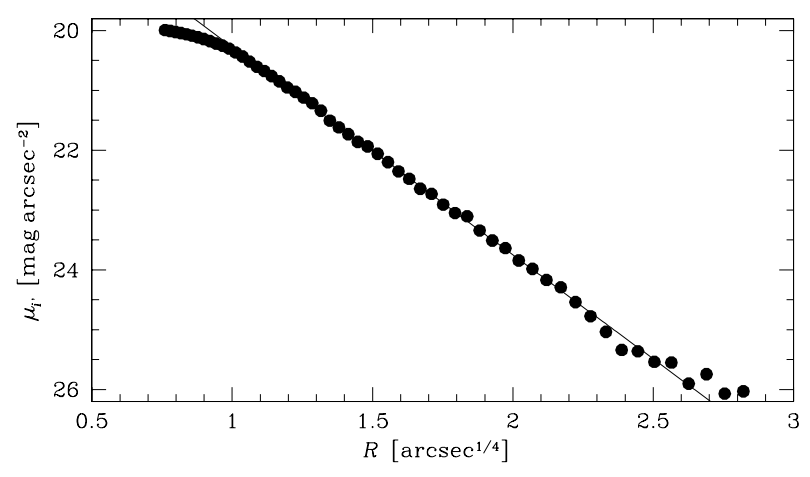

Fig. 11. Surface brightness of the central galaxy in the $i^{\prime}$ band. The solid line represents a $r^{1 / 4}$ (de Vaucouleurs) profile. The size of the symbols represents upper limits of the estimated errors of $\mu_{i^{\prime}}$.

Assuming that such an arc-like feature is indeed a lensed background galaxy, we use the singular isothermal sphere (SIS) model to roughly estimate the cluster mass up to its angular position. We further assume that the arc is located at the Einstein radius, in this case, $22^{\prime \prime}\left(90 h_{70}^{-1} \mathrm{kpc}\right.$ at the cluster distance) from the centre of the brightest cluster galaxy. If the background galaxy has $0.8 \lessgtr z \lesssim 4$, then the mass is $(3.5 \lessgtr M \lessgtr 5.5) \times$ $10^{13} M_{\odot}$. That is roughly 3 to 4 times the total mass obtained with the X-ray observation (see Fig. 6).

The total mass derived by gravitational lensing tends to be different from the mass obtained with X-ray observations (e.g. Allen et al. 2002; Cypriano et al. 2004) for hot and/or disturbed clusters. Moreover, the mass derived from lensing may be higher than the X-ray derived mass due to the mass concentrations along the line-of-sight of the cluster (Metzler et al. 2001). The candidate arc lies at a position where it can indeed be an image of a lensed background object. However, only deeper and higher resolution imaging and/or spectroscopy of the arc itself would confirm its nature.

\section{Summary and conclusions}

In this paper we report on the discovery of $\mathrm{Cl} 2334+48$, a cluster of galaxies in the Zone of Avoidance, from XMM-Newton X-ray images. New photometric and spectroscopic observations of the system are presented. The main results of our analysis of the $\mathrm{X}$-ray and optical data can be summarised as follows.

- $\mathrm{Cl} 2334+48$ is at a redshift of $z=0.271 \pm 0.001$, based on the optical spectrum of the brightest cluster member. The redshift derived from the X-ray spectral fitting is $z=0.263_{-0.010}^{+0.012}$.

- The X-ray emission is consistent with an isothermal ICM with $k T=4.92_{-0.48}^{+0.50} \mathrm{keV}$, gas metallicity of $0.38 \pm 0.12 Z_{\odot}$, and bolometric luminosity of $3.2 \times 10^{44} \mathrm{erg} \mathrm{cm}^{-2} \mathrm{~s}^{-1}$.

- There is no evidence of a strong local $N_{\mathrm{H}}$ absorption in the ICM.

- It has, within $800 h_{70}^{-1} \mathrm{kpc}$, a gas mass of $3.2 \times 10^{13} M_{\odot}$ or $3.5 \times 10^{13} M_{\odot}$, a gas fraction of 0.09 or 0.16 , and a dynamical mass of $3.8 \times 10^{14} M_{\odot}$ or $2.3 \times 10^{14} M_{\odot}$ if either a Sérsic or a $\beta$-model, respectively, is considered for the gas and total mass cumulative profiles. The total virial mass of the cluster within the virial radius of $1.7 h_{70}^{-1} \mathrm{Mpc}$ was determined to be $M_{200}=5 \times 10^{14} M_{\odot}$.

- There is a significant overdensity of galaxies around the brightest cluster object, within the inner $65^{\prime \prime}\left(\sim 270 h_{70}^{-1} \mathrm{kpc}\right)$. Most objects are red, with $g^{\prime}-i^{\prime}>2.5$, as expected if these are members of the cluster.
- The surface brightness of the central galaxy corresponds to a $r^{1 / 4}$ law, indicating that the object is a normal elliptical and not a cD galaxy.

- An arc-like structure, probably due to strong lensing, is seen in both the $g^{\prime}$ and $i^{\prime}$ optical images. This feature is located at $\sim 22^{\prime \prime}\left(90 h_{70}^{-1} \mathrm{kpc}\right)$ from the central galaxy of the cluster. Better images are needed to obtain a secure mass determination of the cluster from the gravitational lensing map.

Although $\mathrm{Cl} 2334+48$ was detected in X-ray in the past (see Sect. 2), its nature was unknown. At a Galactic latitude of $-12^{\circ}$, an optical identification of the cluster, alone, would be difficult. Due to the high sensitivity and good spatial resolution of the XMM-Newton EPIC cameras, such a cluster was easily detected, and we were able to derive its main X-ray properties, as well as its redshift.

The X-ray emission is almost spherically symmetric and the temperature profile has the usual shape (although the error bars are large) observed in relaxed clusters (Figs. 2 and 5). The $\mathrm{X}$-ray derived properties based on hydrostatic equilibrium follow the scaling relations of nearby regular clusters. However, the $\mathrm{X}$-ray peak emission does not coincide with the brightest member galaxy by an offset of $\sim 10^{\prime \prime}\left(40 h_{70}^{-1} \mathrm{kpc}\right.$; see Fig. 2). If this displacement is real, it indicates that this cluster is not quite relaxed and some dynamical event (possibly a sub-cluster merging we may surmise) took place a few Gyrs ago. The central galaxy is not a cD, owing to the lack of an extended stellar envelope, which may be interpreted as a sign of the relative youth of this cluster.

Acknowledgements. We would like to thank G.J.M. Luna for his participation in this discovery. We would also like to thank P. Coté and M. West for obtaining the Keck spectrum of the central galaxy of the cluster, M. Bolte for reducing the spectroscopic data, D. Bortoletto for the help with Figs. 9-11, and the referee, D. Proust, for his suggestions and comments. The INT is operated on the island of La Palma by the Isaac Newton Group in the Spanish Observatorio del Roque de Los Muchachos of the Instituto de Astrofísica de Canarias. R.L.O. acknowledges financial support from the Brazilian agencies FAPESP (grant 03/06861-6) and CAPES (grant BEX0784/04-4), and the Observatoire de Strasbourg. G.B.L.N. acknowledges support from CNPq and CAPES/Cofecub Brazilian-French collaboration. C.M.d.O. and G.B.L.N. would like to thank support from FAPESP through the Thematic Project 01/07342-7.

\section{References}

Adami, C., Ulmer, M. P., Romer, A. K., et al. 2000, ApJS, 131, 391 Allen, S. W., Schmidt, R., \& Fabian, A. C. 2002, MNRAS, 334, L11 Anders, E., \& Grevesse, N. 1989, Geochim. Cosmochim. Acta, 53, 197 Arfken, G. B. 1970, Mathematical methods for physicists, Academic Press, 2nd edn.

Arnaud, M., Pointecouteau, E., \& Pratt, G. W. 2005, A\&A, 441, 893

Balucinska-Church, M., \& McCammon, D. 1992, ApJ, 400, 699

Bardeen, J. M., Bond, J. R., Kaiser, N., \& Szalay, A. S. 1986, ApJ, 304, 15

Bertin, E., \& Arnouts, S. 1996, A\&AS, 117, 393

Cavaliere, A., \& Fusco-Femiano, R. 1976, A\&A, 49, 137

Cypriano, E. S., Sodré, L., Jr., Kneib, J-P., \& Campusano, L. E. 2004, ApJ, 613, 95

Dickey, J. M., \& Lockman, F. J. 1990, ARA\&A, 28, 215

Durret, F., Lima Neto, G. B., \& Forman, W. 2005, A\&A, 432, 809

Ebeling, H., Voges, W., Bohringer, H., et al. 1996, MNRAS, 281, 799

Ebeling, H., Mullis, C. R., \& Tully, R. B. 2002, ApJ, 580, 774

Evrard, A. E., Metzler, C. A., \& Navarro, J. F. 1996, ApJ, 469, 494

Fukugita, M., Hogan, C. J., \& Peebles, P. J. E. 1998, ApJ, 503, 518

Gioia, I., \& Luppino, G. 1994, ApJS, 94, 583

Henry, J. P. 2000, ApJ, 534, 565

Hertz, P., \& Grindlay, J. E. 1988, ApJ, 278, 137 
Hertz, P., \& Grindlay, J. E. 1988, AJ, 96, 233

Hudson, M. J., Smith, R. J., Lucey, J. R., \& Branchini, E. 2004, MNRAS, 352, 61

Kaastra, J. S., \& Mewe, R. 1993, A\&AS, 97, 443

Kolatt, T., Dekel, A., \& Lahav, O. 1995, MNRAS, 275, 797

Kraan-Korteweg, R. C., \& Juraszek, S. 2000, Publ. Astron. Soc. Aust., 17, 6

Liedahl, D. A., Osterheld, A. L., \& Goldstein, W. H. 1995, ApJ, 438, L115

Lima Neto, G. B., Gerbal, D., \& Márquez, I. 1999, MNRAS, 309, 481

Lumb, D. H., Warwick, R. S., Page, M., \& De Luca, A. 2002, A\&A, 389, 93

Lynden-Bell, D., Faber, S. M., Burstein, D., et al. 1988, ApJ, 326, 19

Metzler, C. A., White, M., \& Loken, C. 2001, ApJ, 547, 560

Mieske, S., Hilker, M., \& Infante, L. 2005, A\&A, 438, 103

Monet, D., Bird, A., Canzian, B., et al. 1998, USNO-A V2.0: A Catalog of Astrometric Standards (Flagstaff: US Naval Observatory)

Motch, C., Herent, O., \& Guillout, P. 2003, Astron. Nachr., 324, 61

Motch, C., Barcons, X., Carrera, F., et al. 2006, A\&A, submitted

Nagayama, T., Woudt, P. A., Wakamatsu, K., et al. 2006, MNRAS, 368, 534

Navarro, J., Frenk, C. S., \& White, S. D. M. 1997, ApJ, 490, 493
Nelson, R. F., \& Spencer, R. E. 1988, MNRAS, 234, 1105

Nevalainen, J., Lumb, D., dos Santos, S., et al. 2001, A\&A, 374, 66

Proust, D., Quintana, H., Carrasco, E. R., et al. 2006, A\&A, 447, 133

Radburn-Smith, D. J., Lucey, J. R., Woudt, P. A., Kraan-Korteweg, R. C., \& Watson, F. G. 2006, MNRAS, 369, 1131

Rosati, P., Della Ceca, R., Norman, C., \& Giacconi, R. 1998, ApJ, 492, L21

Rosati, P., Borgani, S., \& Norman, C. 2002, ARA\&A, 40, 539

Schlegel, D. J., Finkbeiner, D. P., \& Davis, M. 1998, ApJ, 500, 525

Sérsic, J. L. 1968, Atlas de Galaxias Australes, Cordoba, Argentina

Tonry, J. L., Blakeslee, J. P., Ajhar, E. A., \& Dressler, A. 2000, ApJ, 530, 625

Voges, W., Aschenbach, B., Boller, Th., et al. 1999, A\&AS, 349, 389

Watson, M. G., Auguères, J.-L., Ballet, J., et al. 2001, A\&A, 365, 51

White, S. D. M., Navarro, J. F., Evrard, A. E., \& Frenk, C. S. 1993, Nature, 366, 429

White, N. E., Giommi, P., \& Angelini, L. 2000, The WGACAT version of the ROSAT PSPC Catalogue, Rev. 1, Laboratory for High Energy Astrophysics (LHEA/NASA), Greenbelt

Yuan, W., McMahon, R. G., Watson, M., et al. 2003, Astron. Nachr., 324, 178 